\title{
Collinear triples in permutations
}

\author{
Liangpan Li
}

go back

full screen

close

quit

\begin{abstract}
Let $\alpha: \mathbb{F}_{q} \rightarrow \mathbb{F}_{q}$ be a permutation and $\Psi(\alpha)$ be the number of collinear triples in the graph of $\alpha$, where $\mathbb{F}_{q}$ denotes a finite field of $q$ elements. When $q$ is odd, Cooper and Solymosi once proved $\Psi(\alpha) \geq(q-1) / 4$ and conjectured the sharp bound should be $\Psi(\alpha) \geq(q-1) / 2$. In this note we confirm this conjecture.
\end{abstract}

Keywords : collinear triple, permutation, Kakeya set

MSC 2000: 11T99

\section{Introduction}

Denote by $\mathbb{F}_{q}$ the finite field of $q$ elements with $q$ odd. Let $\alpha: \mathbb{F}_{q} \rightarrow \mathbb{F}_{q}$ be a permutation and $\Psi(\alpha)$ be the number of collinear triples in

$$
G_{\alpha}=\left\{(i, \alpha(i)): i \in \mathbb{F}_{q}\right\}
$$

the graph of $\alpha$. Cooper and Solymosi [5] once obtained the lower bound

$$
\Psi(\alpha) \geq \frac{q-1}{4},
$$

and conjectured the best one should be

$$
\Psi(\alpha) \geq \frac{q-1}{2} .
$$

Later Cooper [4] showed that the problem of counting collinear triples in a permutation and the finite plane Kakeya problem are intimately connected, and improved (1) slightly to

$$
\Psi(\alpha) \geq \frac{5 q-1}{14}
$$

The main purpose of this note is to indicate that the Cooper-Solymosi conjecture (2) is true. We also mention that Ball [1] has proved (2) in a very nice way. 


\section{Proof}

A subset in $\mathbb{F}_{q}^{2}$ containing a line in each direction is called a Kakeya set. Given the permutation $\alpha$, one can construct a corresponding Kakeya set

$$
K_{\alpha} \doteq L(\infty,(0,0)) \cup \bigcup_{i \in \mathbb{F}_{q}} L(i,(0, \alpha(i)))
$$

where $L(s, x)$ denotes the line in $\mathbb{F}_{q}^{2}$ through $x$ with slope $s$. Writing $\mu_{x}$ for the number of these lines passing through $x$, it follows from the incidence formula of Faber [6] that

$$
\sharp K_{\alpha}=\frac{q(q+1)}{2}+\sum_{x \in K_{\alpha}}\left(\begin{array}{c}
\mu_{x}-1 \\
2
\end{array}\right) .
$$

By duality (cf. [4]), a point $x$ in $K_{\alpha}$ with $\mu_{x} \geq 3$ corresponds to a collinear $\mu_{x}$-tuple in $G_{\alpha}$, and vice versa. Thus denoting by $\Gamma_{\alpha}$ the hypergraph on the vertex set $G_{\alpha}$ whose edges are the maximal collinear subsets of $G_{\alpha}$, (3) turns out to be

$$
\sharp K_{\alpha}=\frac{q(q+1)}{2}+\sum_{e \in E\left(\Gamma_{\alpha}\right)}\left(\begin{array}{c}
|e|-1 \\
2
\end{array}\right) .
$$

By considering

$$
\Psi(\alpha)=\sum_{e \in E\left(\Gamma_{\alpha}\right)}\left(\begin{array}{c}
|e| \\
3
\end{array}\right) \geq \sum_{e \in E\left(\Gamma_{\alpha}\right)}\left(\begin{array}{c}
|e|-1 \\
2
\end{array}\right),
$$

to confirm (2) it suffices to prove

$$
\sharp K_{\alpha} \geq \frac{q(q+1)}{2}+\frac{q-1}{2} .
$$

Coincidentally, for any Kakeya set $K \subset \mathbb{F}_{q}^{2}$, Faber [6] has proved the bound

$$
\sharp K \geq \frac{q(q+1)}{2}+\frac{q}{3},
$$

and conjectured the sharp one should be

$$
\sharp K \geq \frac{q(q+1)}{2}+\frac{q-1}{2} .
$$

Recently, by exploiting the Jamison-Brouwer-Schrijver bound $[3,7]$ on the size of blocking sets in Desarguesian affine planes, (5) was established independently by Blokhuis and Mazzocca [2] and Ball [1]. As an immediate corollary, (4) is true, and so is (2). 
